\title{
Distinct action of the $\alpha$-glucosidase inhibitor miglitol on SGLT3, enteroendocrine cells, and GLP1 secretion
}

\author{
Eun Young Lee, Shuji Kaneko', Promsuk Jutabha², Xilin Zhang, Susumu Seino ${ }^{3}$, \\ Takahito Jomori ${ }^{4}$, Naohiko Anzai ${ }^{2}$ and Takashi Miki \\ Department of Medical Physiology, Graduate School of Medicine, Chiba University, 1-8-1 Inohana, Chuo-ku, \\ Chiba 260-8670, Japan \\ ${ }^{1}$ Department of Molecular Pharmacology, Graduate School of Pharmaceutical Sciences, Kyoto University, \\ Kyoto, Japan \\ ${ }^{2}$ Department of Pharmacology and Toxicology, Dokkyo Medical University School of Medicine, \\ Tochigi 321-0293, Japan \\ ${ }^{3}$ Division of Molecular and Metabolic Medicine, Kobe University Graduate School of Medicine, 7-5-1, \\ Kusunoki-cho, Chuo-ku, Kobe 650-0017, Japan \\ ${ }^{4}$ Drug Development Center, Sanwa Kagaku Kenkyusho Co., Ltd, 35 Higashisotobori-cho, Higashi-ku, \\ Nagoya 461-8631, Japan
}

Correspondence should be addressed to T Miki

Email

tmiki@faculty.chiba-u.jp

\begin{abstract}
Oral ingestion of carbohydrate triggers glucagon-like peptide 1 (GLP1) secretion, but the molecular mechanism remains elusive. By measuring GLP1 concentrations in murine portal vein, we found that the ATP-sensitive $\mathrm{K}^{+}\left(\mathrm{K}_{\mathrm{ATP}}\right)$ channel is not essential for glucose-induced GLP1 secretion from enteroendocrine $L$ cells, while the sodium-glucose co-transporter 1 (SGLT1) is required, at least in the early phase $(5 \mathrm{~min})$ of secretion. By contrast, co-administration of the $\alpha$-glucosidase inhibitor $(\alpha-\mathrm{Gl})$ miglitol plus maltose evoked late-phase secretion in a glucose transporter 2-dependent manner. We found that GLP1 secretion induced by miglitol plus maltose was significantly higher than that by another $\alpha$-GI, acarbose, plus maltose, despite the fact that acarbose inhibits maltase more potently than miglitol. As miglitol activates SGLT3, we compared the effects of miglitol on GLP1 secretion with those of acarbose, which failed to depolarize the Xenopus laevis oocytes expressing human SGLT3. Oral administration of miglitol activated duodenal enterochromaffin (EC) cells as assessed by immunostaining of phosphorylated calcium-calmodulin kinase 2 (phospho-CaMK2). In contrast, acarbose activated much fewer enteroendocrine cells, having only modest phospho-CaMK2 immunoreactivity. Single administration of miglitol triggered no GLP1 secretion, and GLP1 secretion by miglitol plus maltose was significantly attenuated by atropine pretreatment, suggesting regulation via vagal nerve. Thus, while $\alpha$-Gls generally delay carbohydrate absorption and potentiate GLP1 secretion, miglitol also activates duodenal EC cells, possibly via SGLT3, and potentiates GLP1 secretion through the parasympathetic nervous system.
\end{abstract}

Journal of Endocrinology (2015) 224, 205-214 http://joe.endocrinology-journals.org
DOI: 10.1530/JOE-14-0555 Printed in Great Britain

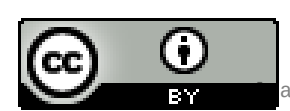

This work is licensed under a Creative Commons Attribution 3.0 Unported License. 


\section{Introduction}

Oral ingestion of nutrients triggers the secretion of gut hormones from various enteroendocrine cells (Ezcurra et al. 2013, Cho et al. 2014). Among these, glucagon-like peptide 1 (GLP1) and glucose-dependent insulinotropic polypeptide (GIP) play a central role in dampening postprandial hyperglycemia after carbohydrate ingestion. Although both GLP1 and GIP are principal incretin hormones that increase insulin secretion, GLP1 is of special interest for its therapeutic potential in type 2 diabetes mellitus (T2DM; Cho et al. 2014). GLP1-targeted anti-diabetic agents comprise GLP1 mimetics and inhibitors of dipeptidyl peptidase 4 that enhance GLP1 action by inhibiting the rapid inactivation of the hormone endogenously secreted from enteroendocrine L cells. The mechanism of carbohydrate-induced GLP1 secretion is therefore critical in the physiology and pathophysiology of glucose homeostasis after meal ingestion. However, the molecular mechanism of carbohydrate-induced GLP1 secretion remains unclear.

Glucose is a potent stimulant of GLP1 secretion. As glucose is the most important energy source and its ambient levels need to be maintained, many cell types in the body are equipped with a glucose-sensing apparatus. These cells include pancreatic $\beta$-cells, glucose-responsive neurons in the hypothalamus, portal glucose sensors, and enteroendocrine cells. Pancreatic $\beta$-cells and glucoseresponsive neurons are electrically excitable cells with a firing rate regulated by extracellular glucose levels. The molecular mechanisms of glucose sensing in these two cell types have been studied extensively. At present, several molecules such as glucose transporter 2 (GLUT2) (Thorens 2003), glucokinase (Matschinsky et al. 2006), and the ATPsensitive $\mathrm{K}^{+}\left(\mathrm{K}_{\mathrm{ATP}}\right)$ channel (Miki \& Seino 2005) have been shown to be critical in glucose sensing in these cells. L cells are morphologically polarized, open-type endocrine cells. $\mathrm{L}$ cells are adjoined with enterocytes in gut mucosa and possess an apical neck with microvilli, in which nutrient in the gut lumen is recognized. While glucose is taken up into enterocytes through sodium-glucose co-transporter 1 (SGLT1) and diffuses into basolateral space through GLUT2, L cells also express both SGLT1 (SLC5A1) and GLUT2 (SLC2A2) (Reimann et al. 2008). In this study, we evaluated the physiological relevance of the $\mathrm{K}_{\mathrm{ATP}}$ channel, SGLT1, and GLUT2 in the glucose sensing of L cells by measuring portal GLP1 concentrations in vivo.

During the course of the study, we noticed that the $\alpha$-glucosidase inhibitor $(\alpha$-GI) miglitol potentiates GLP1 secretion when co-administered with maltose. Inhibitors of a-glucosidase (glucan 1,4-alpha-glucosidase, Enzyme Commission (EC) number; 3.2.1.3) are generally considered to increase postprandial GLP1 levels through delaying digestion and absorption of carbohydrate (Seifarth et al. 1998, Enc et al. 2001). To investigate the mechanism of miglitol on GLP1 secretion, we examined its action on the putative glucose sensor SGLT3, which was originally identified in brain (Diez-Sampedro et al. 2003, Voss et al. 2007).

In this study, we compared two $\alpha$-GIs, miglitol and acarbose, by examining their effects on GLP1 secretion, SGLT3-mediated depolarization in Xenopus laevis oocytes expressing human SGLT3 (hSGLT3), and enteroendocrine cell activation using immunostaining of phosphorylated calcium-calmodulin kinase 2 (phospho-CaMK2).

\section{Materials and methods}

\section{Reagents}

Phlorizin, phloretin, and acarbose were purchased from Wako Pure Chemical Industries, Ltd (Osaka, Japan). Miglitol was provided by Sanwa Kagaku Kenkyusho Co. Ltd (Nagoya, Aichi, Japan).

\section{Animal experiments}

WT and Kir6.2 (Kcnj11)-deficient male mice (Miki et al. 1998) with the same genetic background as that of C57BL/6 mice were used for the study. All animal studies were approved by the Animal Care and Use Committee of Chiba University.

\section{Experiments of GLP1 secretion in vivo}

After 16-h fast, mice were subjected to GLP1 secretion experiments. We utilized two protocols: one for earlyphase (5 min) and the other for late-phase (10 and $30 \mathrm{~min}$ ) GLP1 secretion after sugar loading. For analyzing earlyphase secretion, mice were anesthetized by pentobarbital and isoflurane and were laparotomized to place a gastric gavage in the duodenum. Five minutes after secretagogue ( $20 \mu \mathrm{l} / \mathrm{g}$ body weight) administration through gavage, portal blood $(\sim 600 \mu \mathrm{l})$ was drawn and immediately mixed with EDTA (final 0.15\% w/v) and diprotin A (final, $3 \mathrm{mmol} / \mathrm{l})$. For analyzing late-phase (10 and $30 \mathrm{~min}$ ) secretion, conscious mice were administered secretagogue ( $20 \mu \mathrm{l} / \mathrm{g}$ body weight). Under anesthesia with pentobarbital

Published by Bioscientifica Ltd 
and isoflurane, portal blood $(\sim 600 \mu \mathrm{l})$ was drawn in the same way as described earlier for early-phase sampling. The plasma intact GLP1 concentration was measured using Active GLP1 Assay Kit (Millipore, Billerica, MA, USA).

\section{Histological examination}

Immunostaining of phospho-CaMK2 of gut was carried out under standardized methods. After administration of secretagogues ( $20 \mu \mathrm{l} / \mathrm{g}$ body weight), mice were killed by pentobarbital and isoflurane, perfused, and fixed with $4 \%$ paraformaldehyde. The duodenum was excised and tissues were embedded in O.C.T. Compound (Sakura Finetechnical Co., Tokyo, Japan). Frozen sections of $10 \mu \mathrm{m}$ in thickness were incubated in 1\% goat serum dissolved in PBS-T (PBS containing $0.3 \%$ Tween-20) for $30 \mathrm{~min}$ at room temperature. For double immunofluorescence of phospho-CaMK2 and 5-HT, sections were first stained with a rabbit antipCaMK2 $\left(\mathrm{pT}^{286}\right)$ antibody (1:1000 in PBS-T, Promega) overnight at $4{ }^{\circ} \mathrm{C}$. Incubation with the secondary antibody (goat anti-rabbit IgG antibody conjugated with AlexaFluor 488, 1:500 in PBS, Invitrogen Molecular Probes) was performed for $2 \mathrm{~h}$ at room temperature. Subsequently, the sections were incubated with a mouse anti-5-HT antibody (1:500 in PBS-T, Dako Corp., Carpenteria, CA, USA) overnight at $4{ }^{\circ} \mathrm{C}$. The goat anti-mouse IgG antibody conjugated with AlexaFluor 594 (1:500 in PBS, Invitrogen Molecular Probes) was used thereafter as a secondary antibody. The slides were examined under a FluoView FV10i confocal microscope (Olympus, Tokyo, Japan).

\section{Electrophysiological recording of $X$. laevis oocytes expressing hSGLT3}

As SGLT3 does not transport glucose but carries $\mathrm{Na}^{+}$to elicit membrane depolarization (Diez-Sampedro et al. 2003), the function of SGLT3 was evaluated electrophysiologically in the $X$. laevis oocytes heterologously expressing human SGLT3. hSGLT3 cDNA was inserted into pF1K T7 Flexi vector. The cDNA was linearized by FspI and used to synthesize cRNA using the mMESSAGE mMACHINE T7 RNA polymerase kit (Ambion, Austin, TX, USA). The polyadenylation of cRNA at $3^{\prime}$-end was performed using the Poly(A) tailing kit (Ambion). Small pieces of ovary were removed from $X$. laevis frogs. Follicles were isolated and treated with $1 \mathrm{mg} / \mathrm{ml}$ collagenase (Wako Pure Chemicals) in $\mathrm{Ca}^{2+}$-free solution $(96 \mathrm{mmol} / 1 \mathrm{NaCl}$, $2 \mathrm{mmol} / \mathrm{l} \mathrm{KCl}, 1 \mathrm{mmol} / 1 \mathrm{MgCl}_{2}, 5 \mathrm{mmol} / \mathrm{l} \mathrm{HEPES}, \mathrm{pH} 7.5$ ) for $1.5-2 \mathrm{~h}$ at room temperature. Then, $50 \mathrm{nl}$ of $h S G L T 3$ cRNA $(0.5 \mu \mathrm{g} / \mu \mathrm{l})$ was injected into defolliculated oocytes and maintained at $18{ }^{\circ} \mathrm{C}$ in ND96 buffer $(96 \mathrm{mmol} / \mathrm{l} \mathrm{NaCl}$, $2 \mathrm{mmol} / \mathrm{l} \mathrm{KCl}, 1 \mathrm{mmol} / 1 \mathrm{CaCl}_{2}, 1 \mathrm{mmol} / 1 \mathrm{MgCl}_{2}, 5 \mathrm{mmol} / \mathrm{l}$ HEPES, pH 7.5) supplemented with $2.5 \mathrm{mmol} / 1$ pyruvate and $50 \mu \mathrm{g} / \mathrm{ml}$ gentamicin. Three days after $h S G L T 3$ cRNA injection, the oocytes were used for electrophysiological recording. The oocytes were voltage clamped at a holding potential of $-60 \mathrm{mV}$ using two electrodes connected to an OC-725C amplifier (Warner Instruments, Hamden, CT, USA). To obtain an $\mathrm{I}-\mathrm{V}$ relationship, the voltage was ramped to $+60 \mathrm{mV}$ (1 s duration) at intervals of $1 \mathrm{~min}$.

\section{Statistical analysis}

Results are expressed as means \pm s.E.M. Differences between two groups were assessed using unpaired two-tailed Student's $t$-test unless otherwise specified. Datasets involving more than two groups were assessed by one-way ANOVA. $P<0.05$ was considered statistically significant.

\section{Results}

\section{Evaluation of involvement of SGLT1, GLUT2, and $\mathrm{K}_{\text {ATP }}$ channel in glucose-induced GLP1 secretion}

We first examined GLP1 secretion in vivo by measuring blood glucose levels (Fig. 1A) and plasma GLP1 concentrations (Fig. 1B) in portal vein of anesthetized mice at 5 min after intraduodenal glucose $(2 \mathrm{~g} / \mathrm{kg})$ administration, and found that the GLP1 concentrations were significantly increased $(n=4-8, P<0.001)$. By contrast, when glucose was administered intraperitoneally, there was no increase in GLP1 concentration in spite of the significant rise in blood glucose level (data not shown), which accords with a previous report that glucose is recognized from the luminal side of the gut (Reimann et al. 2008, Cho et al. 2014).

We then evaluated the involvement of SGLT1 and GLUT2 in GLP1 secretion. Intraduodenal administration of the SGLT1 blocker phlorizin with glucose significantly suppressed glucose-induced GLP1 secretion $(n=8$, $P<0.001$ ) (Fig. 1B) along with inhibition of the rise in portal glucose levels $(P<0.001)$ (Fig. 1A). By contrast, administration of the GLUT2 blocker phloretin together with glucose did not affect glucose-induced GLP1 secretion ( $n=4$, not significant (NS)) (Fig. 1B), and the rise in portal glucose was significantly attenuated by phloretin $(P<0.001)$ (Fig. 1A), indicating its effective blockade of glucose efflux from enterocytes into blood. These results suggest that glucose transport through SGLT1 into L cells is critical in GLP1 secretion under this experimental condition. We also assessed the involvement

Published by Bioscientifica Ltd 

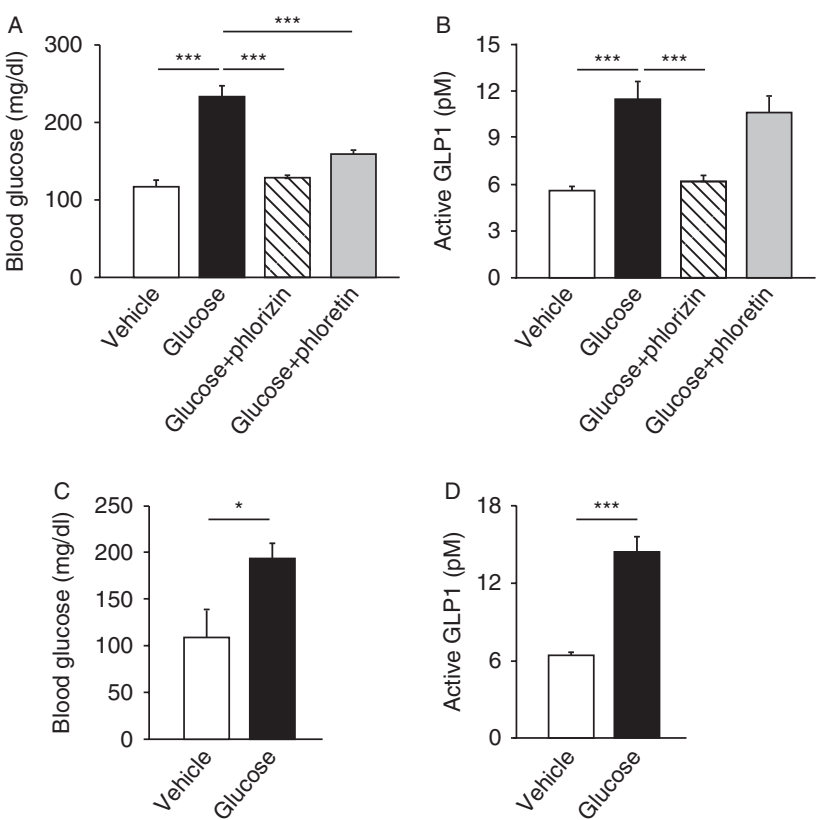

Figure 1

Physiological relevance of SGLT1, GLUT2, and $\mathrm{K}_{\mathrm{ATP}}$ channel in glucoseinduced GLP1 secretion in the early phase. Blood glucose (A) and plasma GLP1 concentrations (B) in portal vein at 5 min after intraduodenal administration of glucose (or vehicle) are shown. Glucose was co-administrated with or without phlorizin $(500 \mathrm{mg} / \mathrm{kg})$ or phloretin $(500 \mathrm{mg} / \mathrm{kg}$ ) to WT mice (A and B). Blood glucose (C) and plasma GLP1 concentrations (D) in portal vein of Kir6.2-deficient mice at $5 \mathrm{~min}$ after intraduodenal glucose administration are also shown. Data are expressed as mean \pm s.E.M. $* P<0.05$ and $* * * P<0.001$.

of the $\mathrm{K}_{\mathrm{ATP}}$ channel in glucose-induced GLP1 secretion using $\mathrm{K}_{\mathrm{ATP}}$ channel-deficient Kir6.2 $2^{-/-}$mice. Intraduodenal glucose administration evoked a significant rise in blood glucose levels $(n=5-8, P<0.05)$ and GLP1 secretion $(P<0.001)$ in Kir6. $2^{-/-}$mice (Fig. $1 C$ and D), similar to that in WT mice, indicating that $\mathrm{K}_{\mathrm{ATP}}$ channels are not essential for GLP secretion.

To investigate the GLP1 secretory mechanism under more physiological conditions, we measured blood glucose levels and GLP1 secretion in unrestrained, conscious mice 30 min after oral administration of glucose $(n=12$, NS) or maltose ( $n=5$, NS) (Fig. 2A and B). No significant increase in GLP1 concentrations by stimulation of either glucose or maltose was found, despite the fact that the portal glucose levels remained elevated at sampling. We then examined the GLP1 secretion at earlier time points (5 and $10 \mathrm{~min}$ ) after oral glucose loading in mice under anesthesia with isoflurane (for $5 \mathrm{~min}$ ) or under awake condition (for $10 \mathrm{~min})$. The portal GLP1 levels were significantly increased at $5 \mathrm{~min}$ after oral glucose loading (15.93 \pm $1.80 \mathrm{pM}, n=4, P<0.01)$. In addition, at $10 \mathrm{~min}$ after oral glucose loading, a significant increase in GLP1 concentrations was apparent $(19.99 \pm 1.94 \mathrm{pM}, n=7, P<0.001)$. These data suggest that the lack of increase at $30 \mathrm{~min}$ after oral glucose loading is probably due to the disappearance of residual luminal glucose at this time point.

We then co-administered the $\alpha$-GI miglitol plus maltose and measured blood glucose levels and GLP1 concentrations at $30 \mathrm{~min}$ (Fig. 2C and D). In this protocol, the blood glucose levels were slightly but significantly increased by administration of miglitol plus maltose ( $n=5-10, P<0.05)$, but the rise was significantly smaller than that by maltose alone $(P<0.01)$. By contrast, co-administration of miglitol significantly increased the GLP1 secretion by maltose $(P<0.001)$. We then evaluated the involvement of SGLT1 and GLUT2 in GLP1 secretion induced by maltose plus miglitol in WT mice. Unlike GLP1 secretion by intraduodenal glucose, the GLP1 secretion by oral maltose plus miglitol was significantly suppressed by phloretin $(n=6, P<0.01)$ but not by phlorizin $(n=15$, NS) (Fig. 2C), suggesting involvement of GLUT2 but not of SGLT1 in this condition. To evaluate involvement of $\mathrm{K}_{\mathrm{ATP}}$ channels in GLP1 secretion in the late phase, we examined the rise in blood glucose levels and the GLP1 secretion by oral administration of maltose plus miglitol in Kir6. $2^{-/-}$ mice (Fig. 2E and F), and found that the GLP1 secretion also occurred in Kir6. $2^{-/-}$mice $(n=6-9, P<0.01)$.

\section{Effect of residual glucose in the gut lumen on GLP1 secretion}

Hypothesizing that GLP1 secretion by maltose plus miglitol might be triggered by residual glucose in the gut lumen, we examined whether blockade of luminal glucose absorption by phlorizin restores the lack of secretion at $30 \mathrm{~min}$. As expected, co-administration of phlorizin (250 mg/kg body weight) with glucose significantly suppressed the rise in blood glucose levels $(n=10$, $P<0.001)$ and increased GLP1 secretion $(P<0.01)$ (Fig. 2G and H). Interestingly, the relatively low dose of phlorizin (125 or $50 \mathrm{mg} / \mathrm{kg}$ body weight) did not suppress the rise in blood glucose levels after glucose load, but evoked significant GLP1 secretion $(n=7, P<0.05$ for $125 \mathrm{mg} / \mathrm{kg}$ phlorizin and $n=13, P<0.001$ for $50 \mathrm{mg} / \mathrm{kg}$ ) in the dose-dependent manner of phlorizin.

\section{Comparison between miglitol and acarbose on GLP1 secretion induced by maltose}

Then, we compared the effects of miglitol and acarbose on GLP1 secretion in mice. Single administration of neither

Published by Bioscientifica Ltd 
A
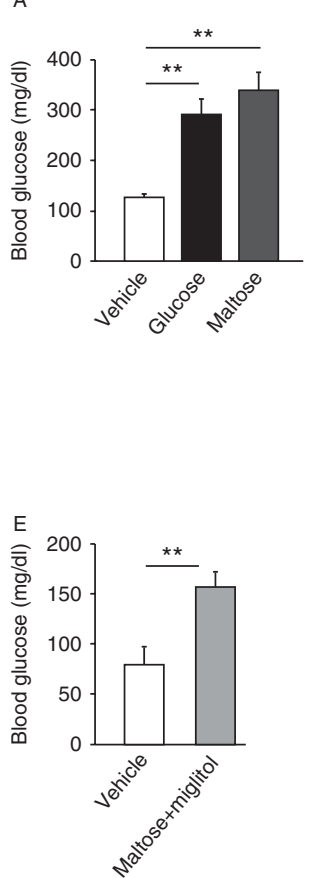

B
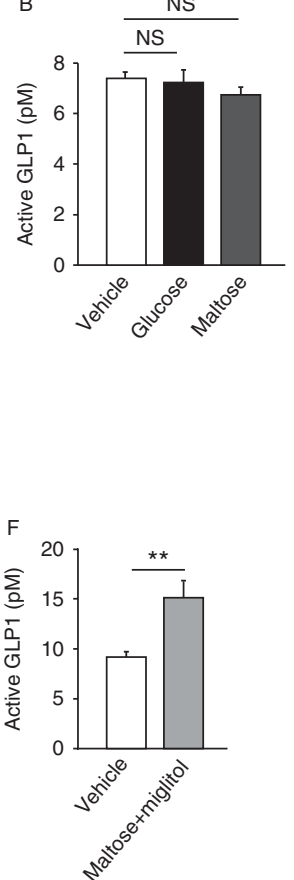
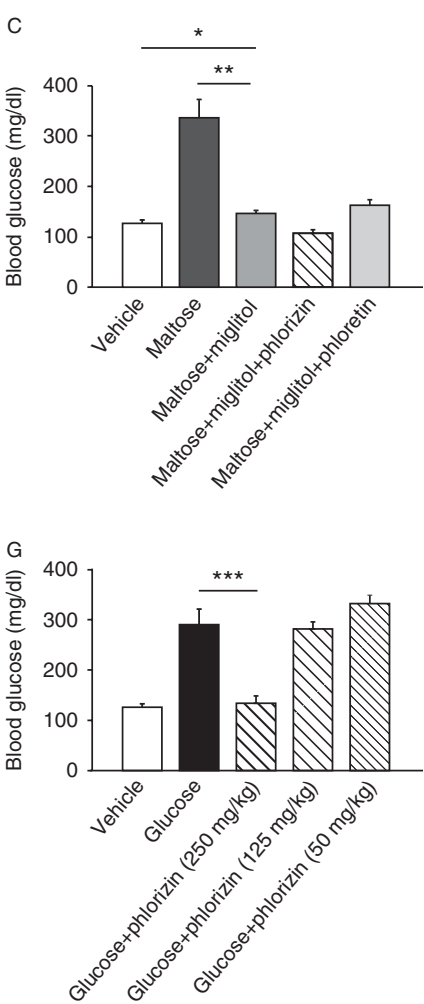

D
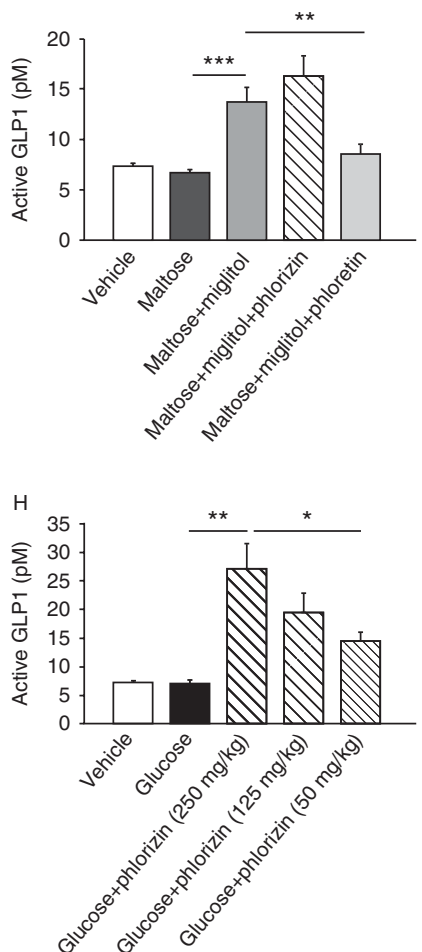

Figure 2

Physiological relevance of SGLT1 and GLUT2 in GLP1 secretion by maltose plus miglitol in the late phase. Blood glucose ( $A$ and $C$ ) and plasma GLP1 concentrations ( $B$ and $D$ ) in the portal vein of awake, WT mice at $30 \mathrm{~min}$ are shown. Data after oral administration of glucose, maltose, or vehicle $(A, B, C$, and $D)$ or of maltose plus miglitol with or without either phlorizin or phloretin (C and D) are shown. Blood glucose (E) and plasma GLP1

miglitol nor acarbose affected blood glucose levels or GLP1 concentrations ( $n=14-16$, NS) (Fig. 3A and B). By contrast, co-administration of miglitol $(P<0.001)$ or acarbose $(P<0.01)$ with maltose both increased GLP1 secretion $(n=9-15)$. Miglitol plus maltose evoked significantly larger secretion than acarbose plus maltose $(n=9-15, P<0.01)$, suggesting that miglitol may play a role in GLP1 secretion through a mechanism other than its $\alpha$-GI activity.

\section{Electrophysiological recording of $X$. laevis oocytes expressing hSGLT3}

In searching for a novel action of miglitol through a mechanism other than $\alpha$-glucosidase inhibition, we investigated its effect on SGLT3, which has been reported to function as a glucose sensor in cholinergic neurons and skeletal muscles (Diez-Sampedro et al. 2003). SGLT3 has also been shown to be expressed in the small intestine (Barcelona et al. 2012). However, the expression of mouse SGLT3 (SGLT3a concentrations (F) in portal vein of $K i r 6.2^{-1-}$ mice at 30 min after oral administration of maltose plus miglitol are shown. Effect of different doses $(250,125$, and $50 \mathrm{mg} / \mathrm{kg})$ of phlorizin on glucose-induced GLP1 secretion is shown (G and H). Data are expressed as mean \pm s.E.M. ${ }^{*} P<0.05,{ }^{*} P<0.01$, and $* * * P<0.001$.

(SLC5A4A) and SGLT3b (SLC5A4B)) in the sub-regions of small intestine has not been examined. We therefore evaluated Sglt3a and Sglt $3 b$ mRNA expressions in duodenum, jejunum, and ileum by RT-PCR in mice (data not shown). Although expression of SGLT3a and SGLT3b was identified in all sub-regions, the expression levels were relatively low in ileum, in which L cells exist most abundantly.

Miglitol shares $\alpha$-glucosidase inhibitory properties with other $\alpha$-GIs such as voglibose and acarbose. As miglitol and acarbose differ structurally (Gloster \& Vocadlo 2012), we expected that acarbose does not activate SGLT3. To ascertain this, we examined the electrophysiological effect of miglitol on SGLT3 using X. laevis oocytes expressing hSGLT3. As reported previously (Barcelona et al. 2012), miglitol depolarized the cells in a $\mathrm{Na}^{+}$-dependent manner (Fig. 4A and B). Administration of glucose or miglitol elicited a sharp and reversible depolarization of hSGLT3expressing oocytes (Fig. 4C). In our protocols, additive or synergic effects of glucose and miglitol were not observed.

Published by Bioscientifica Ltd 

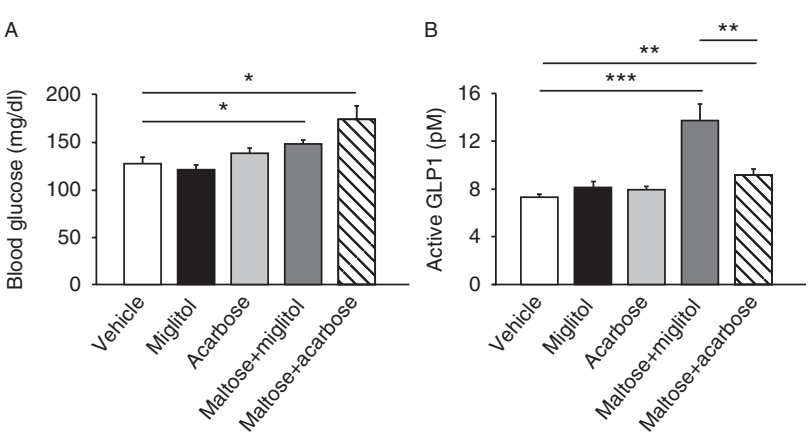

Figure 3

Comparison between miglitol and acarbose of GLP1 secretion induced by maltose. Blood glucose (A) and plasma GLP1 concentrations (B) in the portal vein at $30 \mathrm{~min}$ after oral administration of miglitol, acarbose, maltose plus miglitol, or maltose plus acarbose are shown. Data are expressed as mean \pm S.E.M. ${ }^{*} P<0.05, * * P<0.01$ and $* * * P<0.001$.

In contrast, acarbose failed to depolarize the membrane ( $n=4$ for each group) (Fig. 4D). We therefore compared the effects of miglitol and acarbose on enteroendocrine cell activation.

\section{Histological evaluation of CaMK2 activation in duodenal enteroendocrine cells by glucose, maltose plus miglitol, miglitol, or acarbose}

To evaluate cellular activation in the gut, we examined phosho-CaMK2-positive cells in the duodenum from mice administered (orally, intraperitoneally, or intraduodenally) glucose, maltose plus miglitol, miglitol, acarbose, or vehicle (Fig. 5A, B, C, D, E, and F). As reported previously (Vincent et al. 2011), intraduodenal glucose increased the number of phospho-CaMK2-positive cells (Fig. 5B), but oral vehicle (Fig. 5A) or i.p. glucose administration had no effect (Fig. 5F). The signals of AlexaFluor 594 (conjugated with a secondary antibody against 5-HT) in the cells located inside of intestinal villi are non-specific, as this was similarly detected in the immunostaining without a primary antibody (mouse anti-5-HT antibody) (Supplementary Figure 1, see section on supplementary data given at the end of this article, see details in supplementary data given at the end of the article).

Co-immunostaining of 5-HT and phospho-CaMK2 revealed that most phospho-CaMK2-positive enteroendocrine cells were positive for 5-HT, indicating that these cells were enterochromaffin (EC) cells. In contrast, phosphorylation of CaMK2 was considered not to occur in enteroendocrine L cells either in duodenum or in ileum, as most phospho-CaMK2-positive enteroendocrine cells are EC cells, which constitute a distinct cell population from L cells (Wang et al. 2004).

In addition, oral administration of maltose plus miglitol also evoked phosphorylation of CaMK2 (Fig. 5C). Considering the parallels of CaMK2 phosphorylation in EC cells and GLP1 secretion from L cells by various
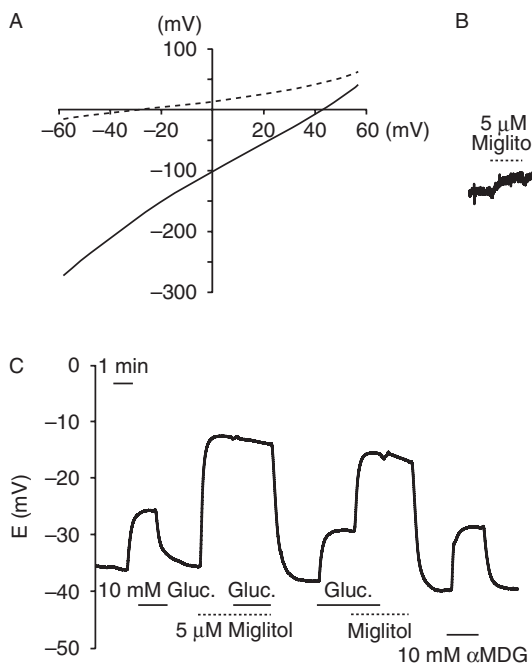

B

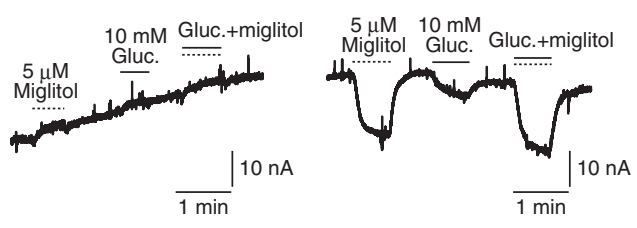

D

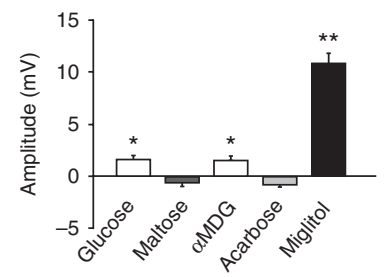

\section{Figure 4}

Electrophysiological recording of Xenopus laevis oocytes expressing hSGLT3. ( $A, B, C$, and D) (A) I-V relationship of the oocytes expressing hSGLT3 in the presence (solid line) or absence (dashed line) of miglitol. (B) A representative recording of whole-cell current under voltage clamp mode in an oocyte expressing hSGLT3 in the absence (left) or presence (right) of $\mathrm{Na}^{+}$in the buffer. 'Gluc.' denotes glucose. (C) A representative recording of the membrane potentials under current clamp mode in an oocyte expressing hSGLT3 in response to glucose and/or miglitol. The SGLT3 agonist $\alpha$-methyl-D-glucopyranoside ( $\alpha$ MDG) was used as a positive control for SGLT3-dependent depolarization. 'Gluc.' denotes glucose. (E) The current changes by several stimuli ( $n=4$ for each). Data are expressed as mean \pm s.E.M. ${ }^{*} P<0.05$ and $* * P<0.01$ (compared with the base line). 
A

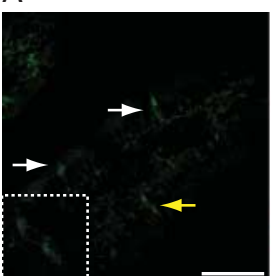

C

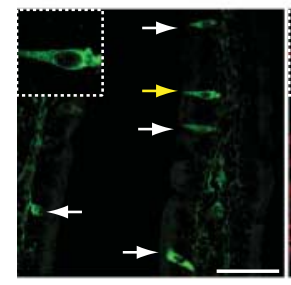

E

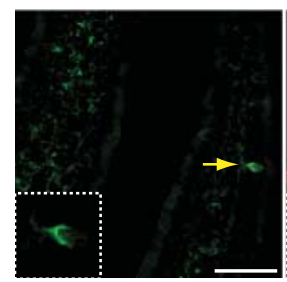

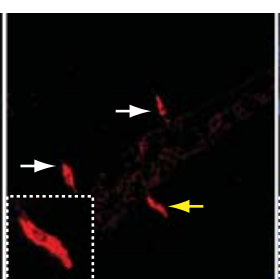
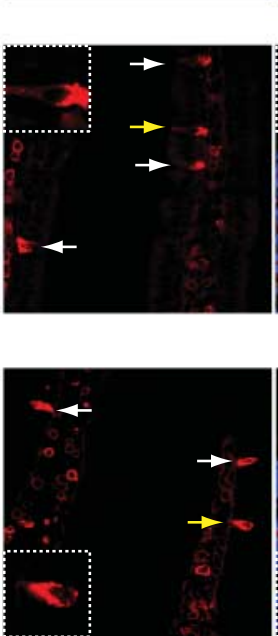
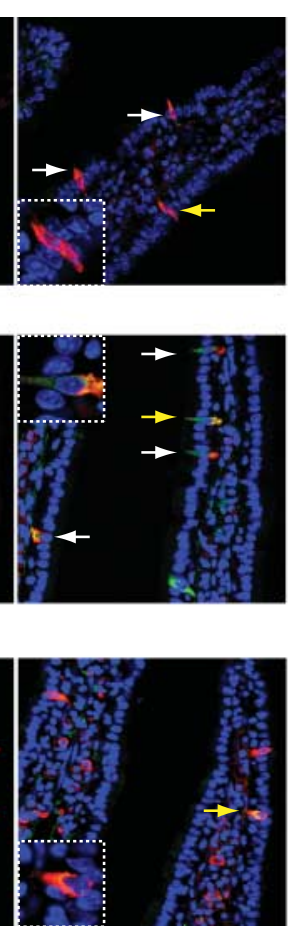

B

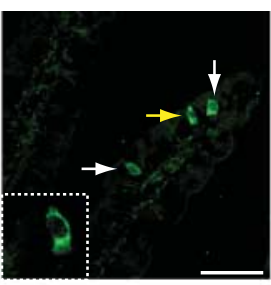

D

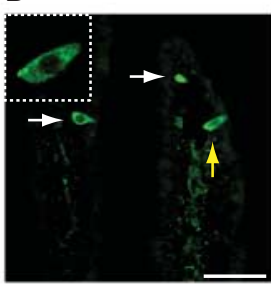

$\mathrm{F}$

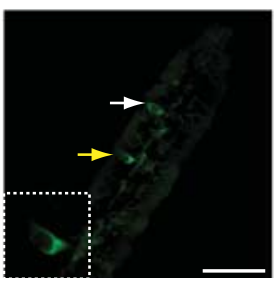

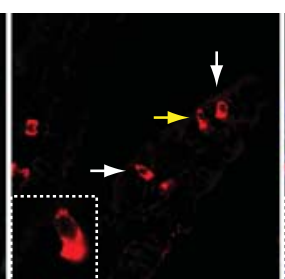
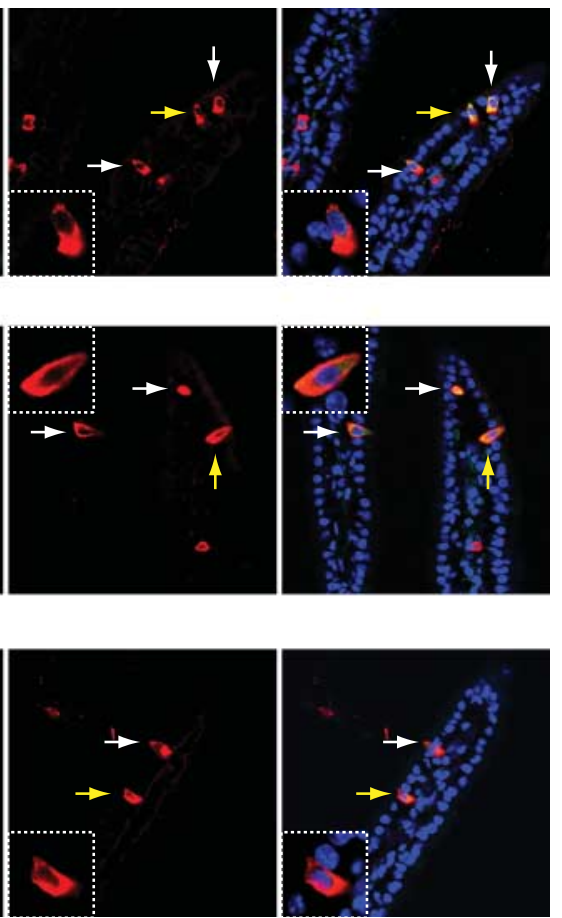

Figure 5

Activation of CaMK2 of duodenal enteroendocrine cells. (A, B, C, D, and E) Immunohistochemistry of phospho-CaMK2 (green) and 5-HT (red) of duodenum is shown. (A) Oral vehicle administration. (B) Intraduodenal glucose administration. (C) Oral maltose plus miglitol administration.

(D) Oral miglitol administration. (E) Oral acarbose administration.

stimuli, we then examined the effects of single administration of either miglitol or acarbose on CaMK2 phosphorylation. Oral administration of miglitol activated duodenal enteroendocrine cells (Fig. 5D). However, in contrast to miglitol, administration of acarbose induced CaMK2 phosphorylation in a lesser number of enteroendocrine cells, with a modest immunoreactivity, to a level comparable to that in mice administered vehicle alone (Fig. 5E).

\section{Evaluation of involvement of vagal nerves in GLP1 secretion by oral administration of maltose plus miglitol}

To clarify autonomic nerve involvement, we pretreated mice with a muscarinic receptor antagonist, atropine, and measured blood glucose levels and GLP1 concentrations (Fig. 6A and B). GLP1 secretion by miglitol plus maltose was significantly attenuated by atropine pretreatment ( $n=6-10, P<0.05)$, indicating parasympathetic nervous system involvement in GLP1 secretion.
(F) Intraperitoneal glucose administration. Insets denote the cells indicated by yellow arrows at a higher magnification. White arrows indicate immunopositive cells. (A, B, C, D, and E) Images of phospho-CaMK2 (left), 5-HT (middle), and their superposition (phospho-CaMK2, 5-HT, and DAPI). Bars indicate $50 \mu \mathrm{m}$.

\section{Discussion}

GLP1 is secreted from enteroendocrine L cells in response to oral nutrient ingestion. Carbohydrates, peptides, and lipids in ingested meal are all known to trigger GLP1 secretion (Cho et al. 2014, Ohlsson et al. 2014). Glucose is a potent secretagogue of GLP1 secretion. Recent studies of primary L cells and the L cell line GLUTag have clarified that L cells express various genes encoding putative glucose sensors, including Sglt1, Glut2, $\mathrm{K}_{\mathrm{ATP}}$ channel subunits (Kir6.2 and Sur1 (Abcc8)), glucokinase, and sweet receptor-related genes (Tas1r2/Tas1r3 and $\alpha$-gastducin) (Cho et al. 2014). However, whether or not SGLT1 and GLUT2 are involved in GLP1 secretion from L cells remains controversial. Some studies have found an essential role of SGLT1 (Reimann et al. 2008, Moriya et al. 2009, Gorboulev et al. 2012); others have emphasized the importance of GLUT2 (Cani et al. 2007, Mace et al. 2012). In this study, we found intraduodenal glucose administration to elicit early-phase (5 min) GLP1

Published by Bioscientifica Ltd 

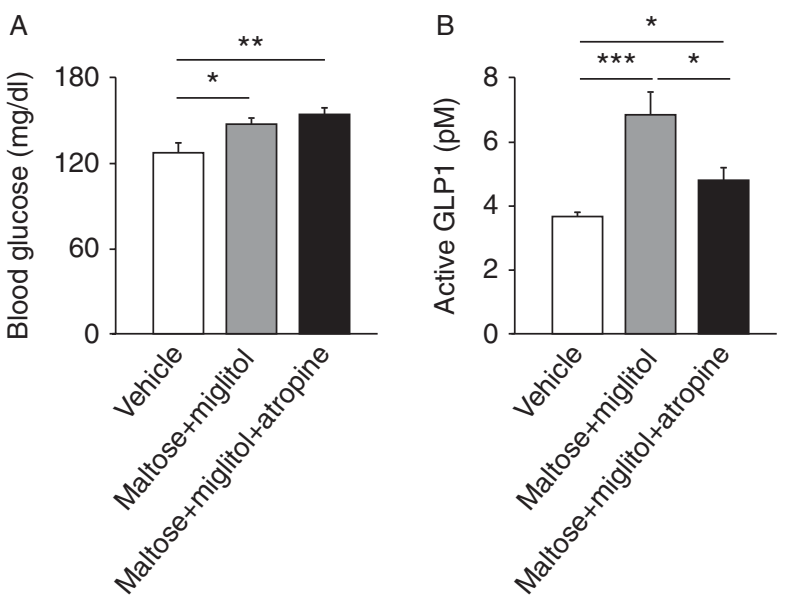

Figure 6

Involvement of parasympathetic nerves in GLP1 secretion. Blood glucose (A) and plasma GLP1 concentrations (B) in the portal vein at $30 \mathrm{~min}$ after oral administration of maltose plus miglitol with or without atropine pretreatment are shown. Data are expressed as mean \pm s.E.M. ${ }^{*} P<0.05$, $* * P<0.01$, and $* * * P<0.001$

secretion, which was blocked by phlorizin (Fig. 1B), while delayed-phase (30 min) GLP1 secretion by maltose plus miglitol was inhibited by phloretin (Fig. 2D). Interestingly, Powell et al. (2013) reported that GLP1 secretion in Sglt1-deficient mice was significantly lower at $5 \mathrm{~min}$ but much higher at $1 \mathrm{~h}$ and later after glucose loading compared with that in WT mice, suggesting a complex contribution of SGLT1 depending on the time course (Powell et al. 2013). Considered together with our present data, we suggest that SGLT1 may be critical for the early phase of glucose-induced GLP1 secretion and that GLUT2 is involved in the late phase, although it remains unknown whether SGLT1 or GLUT2 expressed in L cells participates in this secretion. Regarding the role of $\mathrm{K}_{\mathrm{ATP}}$ channels, several papers have reported that $\mathrm{K}_{\mathrm{ATP}}$ channels in L cells play an important role in GLP1 secretion (Reimann et al. 2008, Mace et al. 2012). However, our results include direct evidence that $\mathrm{K}_{\mathrm{ATP}}$ channels are not essential in GLP1 secretion, although this does not imply that the channels have no role in its regulation.

Co-administration of phlorizin with glucose dose dependently increased GLP1 secretion (Fig. 2H), suggesting that the existence of glucose in the gut lumen is important for triggering the GLP1 secretion in the late phase after glucose load and that this secretion is mediated in a SGLT1-independent manner. This concept is compatible with the clinical observation that $\alpha$-GIs, which delay the hydrolysis of di-, tri-, and polysaccharides, generally potentiate carbohydrate-induced GLP1 secretion
(Hiki et al. 2010, Sakurai et al. 2012). In addition, miglitol was reported to potentiate GLP1 secretion more robustly than acarbose, despite a comparable glucose-lowering effect after meal ingestion (Arakawa et al. 2008). We therefore considered that the effect of miglitol on GLP1 secretion is unlikely to be mediated only by its $\alpha$-GI activity. GLP1 secretion by miglitol plus maltose was larger than that by acarbose plus maltose (Fig. 3B), despite the fact that we applied a much higher dose of acarbose in terms of enzymatic inhibition of maltase (Samulitis et al. 1987). Accordingly, miglitol would seem to retain a potentiating effect on GLP1 secretion through a mechanism other than $\alpha$-glucosidase inhibition.

In searching for a novel mechanism of miglitol action, we evaluated its effect on SGLT3 (Diez-Sampedro et al. 2003). We found that SGLT3 is expressed in all sub-regions of small intestine and to a lesser extent in ileum. Similar to SGLT1, SGLT3 is activated by glucose and elicits $\mathrm{Na}^{+}$-dependent depolarization. However, SGLT3 does not transport glucose (Barcelona et al. 2012) and is therefore considered to be a glucose sensor. SGLT3 has been shown to be activated by several imino-sugars, including 1-deoxynojirimycin (DNJ), N-butyl-1-deoxynojirimycin (miglustat), and $\mathrm{N}$-hydroxyethyl-a-deoxynojirimycin (miglitol) (Diez-Sampedro et al. 2003, Barcelona et al. 2012).

Miglitol and acarbose are structurally different (Gloster \& Vocadlo 2012) and our electrophysiological recordings revealed that miglitol and glucose but not acarbose activate hSGLT3 (Fig. 4D), which led us to compare the effects of miglitol and acarbose on enteroendocrine cell activation. Notably, administration of glucose (intraduodenally) or miglitol (orally) evoked CaMK2 phosphorylation, but acarbose failed to elicit CaMK2 phosphorylation (Fig. 5). Although a single administration of miglitol activated enteroendocrine cells in the duodenum, it failed to increase the GLP1 secretion (Fig. 3B). Miglitol is therefore unlikely to act directly on L cells to trigger the GLP1 secretion.

Nutrient sensing in gut lumen has been reported to play important roles in glucose homeostasis. Especially, jejunum and duodenum are known to play an important role in glucose sensing to regulate glucose production in the liver (Breen et al. 2012, 2013, Marina et al. 2012). Considering that $\mathrm{L}$ cells exist abundantly in the terminal ileum (Tolhurst et al. 2009), there might well be a mechanism linking nutrient sensing in the upper small intestine with GLP1 secretion in the ileum. In addition, our results suggest that the parasympathetic nervous system is probably involved in GLP1 secretion (Fig. 6A and B). This accords with the previous report that

Published by Bioscientifica Ltd 
postprandial GLP1 secretion is mediated through the vagal nervous system in rats (Anini et al. 2002).

Accordingly, we propose a model for GLP1 secretion by maltose plus miglitol as follows: miglitol in the luminal side of the duodenum activates 5-HT-positive EC cells via SGLT3 to trigger the secretion of 5-HT, which activates the parasympathetic nervous system. Simultaneously, miglitol inhibits the hydrolysis of maltose, leading to the increase in the amount of glucose in the ileum, in which L cells exist abundantly. The combination of the existence of luminal glucose in the ileum and parasympathetic nervous input to L cells then evokes GLP1 secretion at late phase (30 min) after oral administration.

Considering the efficacy of GLP1-targeted therapy in the treatment of T2DM, augmentation of GLP1 secretion may be a novel therapeutic target of the disease.

\section{Supplementary data}

This is linked to the online version of the paper at http://dx.doi.org/10.1530/ JOE-14-0555.

\section{Declaration of interest}

The authors declare that there is no conflict of interest that could be perceived as prejudicing the impartiality of the research reported.

\section{Funding}

This work was supported by Research Grants from the Ministry of Education, Science, Sports, and Culture, Japan, and a grant (the 3rd Lilly Incretin Basic Research Grant) from Japan Diabetes Foundation.

\section{Acknowledgements}

The authors thank Dr Toshihiko Iwanaga (Hokkaido University) for his helpful discussion.

\section{References}

Anini Y, Hansotia T \& Brubaker PL 2002 Muscarinic receptors control postprandial release of glucagon-like peptide-1: in vivo and in vitro studies in rats. Endocrinology 143 2420-2426. (doi:10.1210/ endo.143.6.8840)

Arakawa M, Ebato C, Mita T, Fujitani Y, Shimizu T, Watada H, Kawamori R \& Hirose T 2008 Miglitol suppresses the postprandial increase in interleukin 6 and enhances active glucagon-like peptide 1 secretion in viscerally obese subjects. Metabolism 57 1299-1306. (doi:10.1016/ j.metabol.2008.04.027)

Barcelona S, Menegaz D \& Diez-Sampedro A 2012 Mouse SGLT3a generates proton-activated currents but does not transport sugar. American Journal of Physiology. Cell Physiology 302 C1073-C1082. (doi:10.1152/ ajpcell.00436.2011)

Breen DM, Rasmussen BA, Kokorovic A, Wang R, Cheung GW \& Lam TK 2012 Jejunal nutrient sensing is required for duodenal-jejunal bypass surgery to rapidly lower glucose concentrations in uncontrolled diabetes. Nature Medicine 18 950-955. (doi:10.1038/nm.2745)

Breen DM, Rasmussen BA, Cote CD, Jackson VM \& Lam TK 2013 Nutrientsensing mechanisms in the gut as therapeutic targets for diabetes. Diabetes 62 3005-3013. (doi:10.2337/db13-0523)

Cani PD, Holst JJ, Drucker DJ, Delzenne NM, Thorens B, Burcelin R \& Knauf C 2007 GLUT2 and the incretin receptors are involved in glucoseinduced incretin secretion. Molecular and Cellular Endocrinology 276 18-23. (doi:10.1016/j.mce.2007.06.003)

Cho YM, Fujita Y \& Kieffer TJ 2014 Glucagon-like peptide-1: glucose homeostasis and beyond. Annual Review of Physiology 76 535-559. (doi:10.1146/annurev-physiol-021113-170315)

Diez-Sampedro A, Hirayama BA, Osswald C, Gorboulev V, Baumgarten K, Volk C, Wright EM \& Koepsell H 2003 A glucose sensor hiding in a family of transporters. PNAS 100 11753-11758. (doi:10.1073/pnas. 1733027100)

Enc FY, Imeryuz N, Akin L, Turoglu T, Dede F, Haklar G, Tekesin N, Bekiroglu N, Yegen BC, Rehfeld JF et al. 2001 Inhibition of gastric emptying by acarbose is correlated with GLP-1 response and accompanied by CCK release. American Journal of Physiology. Gastrointestinal and Liver Physiology 281 G752-G763.

Ezcurra M, Reimann F, Gribble FM \& Emery E 2013 Molecular mechanisms of incretin hormone secretion. Current Opinion in Pharmacology $\mathbf{1 3}$ 922-927. (doi:10.1016/j.coph.2013.08.013)

Gloster TM \& Vocadlo DJ 2012 Developing inhibitors of glycan processing enzymes as tools for enabling glycobiology. Nature Chemical Biology 8 683-694. (doi:10.1038/nchembio.1029)

Gorboulev V, Schurmann A, Vallon V, Kipp H, Jaschke A, Klessen D, Friedrich A, Scherneck S, Rieg T, Cunard R et al. $2012 \mathrm{Na}(+$ )-D-glucose cotransporter SGLT1 is pivotal for intestinal glucose absorption and glucose-dependent incretin secretion. Diabetes 61 187-196. (doi:10.2337/db11-1029)

Hiki M, Shimada K, Kiyanagi T, Fukao K, Hirose K, Ohsaka H, Fukushima Y, Kume A, Matsumori R, Sumiyoshi K et al. 2010 Single administration of $\alpha$-glucosidase inhibitors on endothelial function and incretin secretion in diabetic patients with coronary artery disease. Circulation Journal $\mathbf{7 4}$ 1471-1478. (doi:10.1253/circj.CJ-10-0013)

Mace OJ, Schindler M \& Patel S 2012 The regulation of K- and L cell activity by GLUT2 and the calcium-sensing receptor CasR in rat small intestine. Journal of Physiology 590 2917-2936. (doi:10.1113/jphysiol. 2011.223800)

Marina AL, Utzschneider KM, Wright LA, Montgomery BK, Marcovina SM \& Kahn SE 2012 Colesevelam improves oral but not intravenous glucose tolerance by a mechanism independent of insulin sensitivity and $\beta$-cell function. Diabetes Care 35 1119-1125. (doi:10.2337/ dc11-2050)

Matschinsky FM, Magnuson MA, Zelent D, Jetton TL, Doliba N, Han Y, Taub R \& Grimsby J 2006 The network of glucokinase-expressing cells in glucose homeostasis and the potential of glucokinase activators for diabetes therapy. Diabetes 55 1-12. (doi:10.2337/diabetes.55.01. 06.db05-0926)

Miki T \& Seino S 2005 Roles of $\mathrm{K}_{\mathrm{ATP}}$ channels as metabolic sensors in acute metabolic changes. Journal of Molecular and Cellular Cardiology 38 917-925. (doi:10.1016/j.yjmcc.2004.11.019)

Miki T, Nagashima K, Tashiro F, Kotake K, Yoshitomi H, Tamamoto A, Gonoi T, Iwanaga T, Miyazaki J \& Seino S 1998 Defective insulin secretion and enhanced insulin action in $\mathrm{K}_{\mathrm{ATP}}$ channel-deficient mice. PNAS 95 10402-10406. (doi:10.1073/pnas.95.18.10402)

Moriya R, Shirakura T, Ito J, Mashiko S \& Seo T 2009 Activation of sodium-glucose cotransporter 1 ameliorates hyperglycemia by mediating incretin secretion in mice. American Journal of Physiology. Endocrinology and Metabolism 297 E1358-E1365. (doi:10.1152/ajpendo. 00412.2009)

Ohlsson L, Kohan AB, Tso P \& Ahrén B 2014 GLP-1 released to the mesenteric lymph duct in mice: effects of glucose and fat. Regulatory Peptides 189 40-45. (doi:10.1016/j.regpep.2014.02.001) 
Powell DR, Smith M, Greer J, Harris A, Zhao S, DaCosta C, Mseeh F, Shadoan MK, Sands A, Zambrowicz B et al. 2013 LX4211 increases serum glucagon-like peptide 1 and peptide YY levels by reducing sodium/glucose cotransporter 1 (SGLT1)-mediated absorption of intestinal glucose. Journal of Pharmacology and Experimental Therapeutics 345 250-259. (doi:10.1124/jpet.113.203364)

Reimann F, Habib AM, Tolhurst G, Parker HE, Rogers GJ \& Gribble FM 2008 Glucose sensing in L cells: a primary cell study. Cell Metabolism $\mathbf{8}$ 532-539. (doi:10.1016/j.cmet.2008.11.002)

Sakurai K, Lee EY, Morita A, Kimura S, Kawamura H, Kasamatsu A, Shiiba M, Yabe D, Yokote K \& Miki T 2012 Glucagon-like peptide-1 secretion by direct stimulation of $\mathrm{L}$ cells with luminal sugar vs non-nutritive sweetener. Journal of Diabetes Investigation 3 156-163. (doi:10.1111/ j.2040-1124.2011.00163.x)

Samulitis BK, Goda T, Lee SM \& Koldovsky O 1987 Inhibitory mechanism of acarbose and 1-deoxynojirimycin derivatives on carbohydrases in rat small intestine. Drugs Under Experimental and Clinical Research 13 517-524.
Seifarth C, Bergmann J, Holst JJ, Ritzel R, Schmiegel W \& Nauck MA 1998 Prolonged and enhanced secretion of glucagon-like peptide 1 (7-36 amide) after oral sucrose due to $\alpha$-glucosidase inhibition (acarbose) in type 2 diabetic patients. Diabetic Medicine 15 485-491. (doi:10.1002/ (SICI) 1096-9136(199806)15:6 < 485::AID-DIA610>3.0.CO;2-Y)

Thorens B 2003 A gene knockout approach in mice to identify glucose sensors controlling glucose homeostasis. Pflügers Archiv 445 482-490.

Tolhurst G, Reimann F \& Gribble FM 2009 Nutritional regulation of glucagon-like peptide-1 secretion. Journal of Physiology 587 27-32. (doi:10.1113/jphysiol.2008.164012)

Vincent KM, Sharp JW \& Raybould HE 2011 Intestinal glucose-induced calcium-calmodulin kinase signaling in the gut-brain axis in awake rats. Neurogastroenterology and Motility 23 e282-e293. (doi:10.1111/ j.1365-2982.2011.01673.x)

Voss AA, Diez-Sampedro A, Hirayama BA, Loo DD \& Wright EM 2007 Imino sugars are potent agonists of the human glucose sensor SGLT3. Molecular Pharmacology 71 628-634. (doi:10.1124/mol.106. 030288)

Received in final form 1 December 2014

Accepted 8 December 2014

Accepted Preprint published online 8 December 2014 http://joe.endocrinology-journals.org DOI: 10.1530/JOE-14-0555 (c) 2015 The authors Printed in Great Britain
Published by Bioscientifica Ltd 